Acta Crystallographica Section F

Structural Biology

and Crystallization

Communications

ISSN 1744-3091

\section{addenda and errata}

\title{
Crystallization and preliminary X-ray diffraction analysis of the high molecular weight ketoacyl reductase FabG4 complexed with NADH. Corrigendum
}

\author{
Debajyoti Dutta, Sudipta Bhattacharyya and Amit Kumar \\ Das*
}

Department of Biotechnology, Indian Institute of Technology, Kharagpur, Kharagpur 721302 , India

Correspondence e-mail: amitk@hijli.iitkgp.ernet.in

In the article by Dutta et al. [(2012) Acta Cryst. F68, 786-789] two citations were given erroneously. These are now corrected.

In the article by Dutta et al. (2012) the citations Gurvitz et al. (2009) and Sharma et al. (2010) were cited erroneously. The correct citations are as follows: Gurvitz et al. (2009) proved the putative function of FabG4 by complementation mutation in yeast and Sharma et al. (2010) showed that FabG4 expresses in Mycobacterium tuberculosis cell in the presence of a sub-inhibitory concentration of streptomycin.

\section{References}

Dutta, D., Bhattacharyya, S. \& Das, A. K. (2012). Acta Cryst. F68, 786-789. Gurvitz, A. (2009). Mol. Genet. Genomics, 282, 407-416.

Sharma, P., Kumar, B., Singhal, N., Katoch, V. M., Venkatesan, K., Chauhan, D. S. \& Bisht, D. (2010). Indian J. Med. Res. 132, 400-408. 